\title{
Indirect dark matter searches in the dwarf satellite galaxy Ursa Major II with the MAGIC Telescopes
}

MAGIC Collaboration: M. L. Ahnen ${ }^{1}$ S. Ansoldi ${ }^{2,20}$

L. A. Antonelli ${ }^{3}$ C. Arcaro $^{4}$ D. Baack ${ }^{5}$ A. Babić ${ }^{6}$ B. Banerjee

P. Bangale ${ }^{8}$ U. Barres de Almeida ${ }^{8,9}$ J. A. Barrio ${ }^{10}$ J. Becerra González $^{11}$ W. Bednarek ${ }^{12}$ E. Bernardini ${ }^{4,13,23}$ R. Ch. Berse ${ }^{5}$

A. Berti ${ }^{2,24}$ W. Bhattacharyya ${ }^{13}$ A. Biland ${ }^{1}$ O. Blanch $^{14}$

G. Bonnoli ${ }^{15}$ R. Carosi $^{15}$ A. Carosi $^{3}$ G. Ceribella ${ }^{8}$ A. Chatterjee ${ }^{7}$

S. M. Colak $^{14}$ P. Colin ${ }^{8}$ E. Colombo ${ }^{11}$ J. L. Contreras ${ }^{10}$

J. Cortina ${ }^{14}$ S. Covino ${ }^{3}$ P. Cumani ${ }^{14}$ P. Da Vela ${ }^{15}$ F. Dazzi ${ }^{3}$

A. De Angelis ${ }^{4}$ B. De Lotto ${ }^{2}$ M. Delfino ${ }^{14,25}$ J. Delgado ${ }^{14}$ F. Di Pierro $^{4}$ A. Domínguez ${ }^{10}$ D. Dominis Prester ${ }^{6}$ D. Dorner ${ }^{16}$

M. Doro ${ }^{4}$ S. Einecke ${ }^{5}$ D. Elsaesser ${ }^{5}$ V. Fallah Ramazani ${ }^{17}$

A. Fernández-Barral ${ }^{14}$ D. Fidalgo ${ }^{10}$ M. V. Fonseca ${ }^{10}$ L. Font ${ }^{18}$

C. Fruck ${ }^{8}$ D. Galindo ${ }^{19}$ R. J. García López ${ }^{11}$ M. Garczarczyk ${ }^{13}$

M. Gaug ${ }^{18}$ P. Giammaria*3 N. Godinović ${ }^{6}$ D. Gora ${ }^{13}$

D. Guberman ${ }^{14}$ D. Hadasch ${ }^{20}$ A. Hahn ${ }^{8}$ T. Hassan $^{14}$

M. Hayashida ${ }^{20}$ J. Herrera ${ }^{11}$ J. Hose $^{8}$ D. Hrupec ${ }^{6}$ K. Ishio ${ }^{8}$

Y. Konno ${ }^{20}$ H. Kubo ${ }^{20}$ J. Kushida ${ }^{20}$ D. Kuveždić ${ }^{6}$ D. Lelas ${ }^{6}$

E. Lindfors ${ }^{17} \mathrm{~S}$. Lombardi ${ }^{* 3} \mathrm{~F}$. Longo ${ }^{2,24} \mathrm{M}$. López ${ }^{10}$ C. Maggio ${ }^{18}$

P. Majumdar ${ }^{7} \mathrm{M}$. Makariev ${ }^{21}$ G. Maneva ${ }^{21} \mathrm{M}$. Manganaro ${ }^{11}$

K. Mannheim ${ }^{16}$ L. Maraschi ${ }^{3}$ M. Mariotti ${ }^{4}$ M. Martínez ${ }^{14}$

S. Masuda ${ }^{20}$ D. Mazin ${ }^{8,20}$ K. Mielke ${ }^{5}$ M. Minev ${ }^{21}$

J. M. Miranda ${ }^{15}$ R. Mirzoyan ${ }^{8}$ A. Moralejo ${ }^{14}$ V. Moreno ${ }^{18}$

E. Moretti ${ }^{8}$ T. Nagayoshi ${ }^{20}$ V. Neustroev ${ }^{17}$ A. Niedzwiecki ${ }^{12}$

M. Nievas Rosillo ${ }^{10}$ C. Nigro ${ }^{13}$ K. Nilsson ${ }^{17}$ D. Ninci ${ }^{14}$

K. Nishijima ${ }^{20}$ K. Noda ${ }^{14}$ L. Nogués ${ }^{14}$ S. Paiano ${ }^{4}$ J. Palacio*14

D. Paneque ${ }^{8}$ R. Paoletti $i^{15}$ J. M. Paredes ${ }^{19}$ G. Pedaletti ${ }^{13}$

M. Peresano ${ }^{2}$ M. Persic ${ }^{2,26}$ P. G. Prada Moroni ${ }^{22}$ E. Prandini ${ }^{4}$

I. Puljak ${ }^{6}$ J. R. Garcia ${ }^{8}$ I. Reichardt ${ }^{4}$ W. Rhode ${ }^{5}$ M. Ribó ${ }^{19}$

J. Rico ${ }^{14}$ C. Righi ${ }^{3}$ A. Rugliancich ${ }^{15}$ T. Saito ${ }^{20}$ K. Satalecka ${ }^{13}$ 


\section{T. Schweizer ${ }^{8}$ J. Sitarek ${ }^{12,20}$ I. Šnidarić ${ }^{6}$ D. Sobczynska ${ }^{12}$ \\ A. Stamerra ${ }^{3}$ M. Strzys ${ }^{8}$ T. Surić ${ }^{6}$ M. Takahashi ${ }^{20}$ L. Takalo ${ }^{17}$ \\ F. Tavecchio ${ }^{3}$ P. Temnikov ${ }^{21}$ T. Terzić ${ }^{6}$ M. Teshima ${ }^{8,20}$ \\ N. Torres-Albà ${ }^{19}$ A. Treves $^{2}$ S. Tsujimoto ${ }^{20}$ G. Vanzo $^{11}$ \\ M. Vazquez Acosta*11 I. Vovk ${ }^{8}$ J. E. Ward ${ }^{14}$ M. Will ${ }^{8}$ D. Zarić ${ }^{6}$}

${ }^{1}$ ETH Zurich, CH-8093 Zurich, Switzerland

${ }^{2}$ Università di Udine, and INFN Trieste, I-33100 Udine, Italy

${ }^{3}$ National Institute for Astrophysics (INAF), I-00136 Rome, Italy

${ }^{4}$ Università di Padova and INFN, I-35131 Padova, Italy

${ }^{5}$ Technische Universität Dortmund, D-44221 Dortmund, Germany

${ }^{6}$ Croatian MAGIC Consortium: University of Rijeka, 51000 Rijeka, University of Split FESB, 21000 Split, University of Zagreb - FER, 10000 Zagreb, University of Osijek, 31000 Osijek and Rudjer Boskovic Institute, 10000 Zagreb, Croatia.

${ }^{7}$ Saha Institute of Nuclear Physics, HBNI, 1/AF Bidhannagar, Salt Lake, Sector-1, Kolkata 700064, India

${ }^{8}$ Max-Planck-Institut für Physik, D-80805 München, Germany

${ }^{9}$ now at Centro Brasileiro de Pesquisas Físicas (CBPF), 22290-180 URCA, Rio de Janeiro (RJ), Brasil

${ }^{10}$ Unidad de Partículas y Cosmología (UPARCOS), Universidad Complutense, E-28040 Madrid, Spain

${ }^{11}$ Inst. de Astrofísica de Canarias, E-38200 La Laguna, and Universidad de La Laguna, Dpto. Astrofísica, E-38206 La Laguna, Tenerife, Spain

${ }^{12}$ University of Łódź, Department of Astrophysics, PL-90236 Łódź, Poland

${ }^{13}$ Deutsches Elektronen-Synchrotron (DESY), D-15738 Zeuthen, Germany

${ }^{14}$ Institut de Física d'Altes Energies (IFAE), The Barcelona Institute of Science and Technology (BIST), E-08193 Bellaterra (Barcelona), Spain

${ }^{15}$ Università di Siena and INFN Pisa, I-53100 Siena, Italy

${ }^{16}$ Universität Würzburg, D-97074 Würzburg, Germany

${ }^{17}$ Finnish MAGIC Consortium: Tuorla Observatory and Finnish Centre of Astronomy with ESO (FINCA), University of Turku, Vaisalantie 20, FI-21500 Piikkiö, Astronomy Division, University of Oulu, FIN-90014 University of Oulu, Finland

${ }^{18}$ Departament de Física, and CERES-IEEC, Universitat Autónoma de Barcelona, E-08193 Bellaterra, Spain

${ }^{19}$ Universitat de Barcelona, ICC, IEEC-UB, E-08028 Barcelona, Spain

${ }^{20}$ Japanese MAGIC Consortium: ICRR, The University of Tokyo, 277-8582 Chiba, Japan; Department of Physics, Kyoto University, 606-8502 Kyoto, Japan; Tokai University, 2591292 Kanagawa, Japan; The University of Tokushima, 770-8502 Tokushima, Japan

${ }^{21}$ Inst. for Nucl. Research and Nucl. Energy, Bulgarian Academy of Sciences, BG-1784 Sofia, Bulgaria

${ }^{22}$ Università di Pisa, and INFN Pisa, I-56126 Pisa, Italy

${ }^{23}$ Humboldt University of Berlin, Institut für Physik D-12489 Berlin Germany

${ }^{24}$ also at Dipartimento di Fisica, Università di Trieste, I-34127 Trieste, Italy

${ }^{25}$ also at Port d'Informació Científica (PIC) E-08193 Bellaterra (Barcelona) Spain 
${ }^{26}$ also at INAF-Trieste and Dept. of Physics \& Astronomy, University of Bologna

*Corresponding authors

E-mail: paola.giammaria@oa-roma.inaf.it, saverio.lombardi@oa-roma.inaf.it, jpalacio@ifae.es, monica.vazquez.acosta@cern.ch

Abstract. The dwarf spheroidal galaxy Ursa Major II (UMaII) is believed to be one of the most dark-matter dominated systems among the Milky Way satellites and represents a suitable target for indirect dark matter (DM) searches. The MAGIC telescopes carried out a deep observation campaign on UMaII between 2014 and 2016, collecting almost one hundred hours of good-quality data. This campaign enlarges the pool of DM targets observed at very high energy $(\mathrm{E} \gtrsim 50 \mathrm{GeV})$ in search for signatures of $\mathrm{DM}$ annihilation in the wide mass range between $\sim 100 \mathrm{GeV}$ and $\sim 100 \mathrm{TeV}$. To this end, the data are analyzed with the full likelihood analysis, a method based on the exploitation of the spectral information of the recorded events for an optimal sensitivity to the explored DM models. We obtain constraints on the annihilation cross-section for different channels that are among the most robust and stringent achieved so far at the $\mathrm{TeV}$ mass scale from observations of dwarf satellite galaxies.

Keywords: dark matter, dwarf spheroidal satellite galaxies, indirect searches, Imaging Air Cherenkov Telescopes, Ursa Major II 


\section{Contents}

1 Introduction $\quad 1$

2 Expected gamma-ray flux from the DM halo of UMaII 3

3 MAGIC and the UMaII observation campaign 4

4 Standard data reduction and results 4

$5 \quad$ Full likelihood analysis method $\quad 6$

6 Results on dark matter annihilation models $\quad 8$

$\begin{array}{lll}7 & \text { Summary and Conclusions } & 10\end{array}$

A DONUT Monte Carlo method 15

\section{Introduction}

Compelling evidence for a large ( $\sim 85 \%)$, dark, non-baryonic and non-relativistic (i.e. "cold") component of the matter density of the Universe arises at all astrophysical scales [1]. We infer its existence from the observations of gravitational effects on galaxies [2,3], galaxy clusters [4-6] and from the anisotropies of the Cosmic Microwave Background [7]. Despite the intensive and multi-approach efforts over the past decades, the nature of dark matter (DM) is still unknown and represents a paramount open issue of modern fundamental Physics and Astrophysics [8]. A particularly well-motivated and widely considered class of cold DM particle candidates is the so-called Weakly Interacting Massive Particle (WIMP [9]). WIMPs spontaneously arise in many Standard Model (SM) extensions (most notably Supersymmetry [10]), have interaction cross-sections typical of the weak scale and a mass in the range between $\sim 10 \mathrm{GeV}$ and tens of $\mathrm{TeV}$, and naturally provide the observed relic density (a fact popularly known as the "WIMP miracle" [11]).

Among different experimental approaches aimed at shedding light on DM nature [1217], indirect searches [18] look for SM particles (i.e. photons, cosmic rays, and neutrinos) produced by DM annihilation or decay processes in DM over-dense astrophysical regions. Due to their complementarity in terms of energy coverage and sensitivity, the spaceborne and ground-based gamma-ray instruments - such as the imaging atmospheric Cherenkov telescopes [19] (IACTs) - operate in synergy in order to scan the wide allowed WIMPs mass range $(\sim 10 \mathrm{GeV}-\sim 100 \mathrm{TeV})$, searching for a flux of gamma rays traced back to DM sources. In this respect, ground-based observations at very high energy (VHE, $\mathrm{E} \gtrsim 50 \mathrm{GeV}$ ) are of major relevance in order to access the complementary parameter space of heavier (and wellmotivated) DM masses with respect to the ones probed by spaceborne instruments at high energy (HE, E > $100 \mathrm{MeV}$ ), as recently shown in the first ever joint DM analysis between Fermi-LAT and MAGIC [20]. Furthermore, the current status of experimental searches seems to strengthen the motivation for WIMPs with masses at the TeV scale or above [21]. This is the mass range where IACTs provide the best sensitivity among all gamma-ray instruments, 
making this class of detectors particularly suited for indirect DM searches in the WIMP scenario.

Indirect DM signatures are expected to be observable in different classes of astrophysical objects, such as the Galactic Center (GC) and Galactic Halo (GH) regions [22], galaxy clusters [23], and dwarf spheroidal satellite galaxies (dSphs) of the Milky Way [24, 25]. Over the last decade, all these classes of targets have been observed at VHE by the current generation of IACTs [26](H.E.S.S. [27, 28], MAGIC [29-31], and VERITAS [32, 33]), so far with no hints of DM signals. Nevertheless, stringent constraints to DM particle models in the $\mathrm{TeV}$ mass range have been set from these observations [20, 27].

The dSph satellites of the Milky Way are among the best-suited targets for indirect DM searches to be observed by gamma-ray instruments (detailed reviews can be found e.g. in $[34,35])$. So far, about thirty among faint and ultra-faint dwarf satellites have been identified by past (e.g. SDSS [36]) and current (e.g. DES [37], Pan-STARRS [38]) deep optical sky surveys, and their number is expected to increase in the next years thanks to the on-going and future optical surveys [39]. This circumstance is of utmost importance for indirect DM searches since new discovered objects may show outstanding features and completely change our current DM detection prospects or capabilities to constrain models.

Ursa Major II [40] (UMaII) is believed to be one of the most DM dominated ultrafaint dSphs, with a Mass-to-Light ratio $\mathrm{M} / \mathrm{L} \sim 4000_{-2000}^{+3700} M_{\odot} / L_{\odot}$ [41]. It has an absolute magnitude of $M_{V} \sim-3.8$, and a distance of $\sim 30 \mathrm{kpc}$ (at RA (J2000) $=8^{h} 51^{\prime} 30.0^{\prime \prime}$ and Dec $\left.(\mathrm{J} 2000)=+63^{\circ} 07^{\prime} 48^{\prime \prime}\right)$. According to kinematic studies [42], the maximum containment angle of DM emission is $\theta_{\max } \simeq 0.53^{\circ 1}$. Therefore, UMaII is an extended source compared to the typical IACT point spread function $\left(\mathrm{PSF} \sim 0.1^{\circ}\right.$ ). This required special care for its observation with MAGIC and for the subsequent data analysis.

The MAGIC telescopes are carrying on deep campaigns for indirect DM searches on several selected sky regions [43]. The diversification of targets is the optimal observational strategy pursued by MAGIC with the aim of reducing the uncertainties and biases in the selection of targets for indirect DM searches. The ultimate goal is to enhance the chances of positive detection and, in case of no hints of DM signal, to achieve the most robust and stringest limits at the $\mathrm{TeV}$ DM mass scale by means of the combination of results coming from different target observations. In this respect, the observation of UMaII belongs to a deep multi-year observation program on dSphs. Thanks to it, MAGIC already provided remarkable limits on DM particle models in the TeV mass range with the deep survey of Segue $1 \mathrm{dSph}$ [30]. In this paper we present the results achieved by means of optimal DM analysis methods of the data taken by MAGIC in a two-years campaign on UMaII.

The rest of the paper is organized as follows: Section 2 provides details on the expected gamma-ray flux from the DM halo of UMaII. Section 3 introduces the MAGIC telescopes and the UMaII observation campaign considered in this study. The standard MAGIC data reduction procedure and results are reported in Section 4. Then, in Section 5, the full likelihood analysis method used to analyze the data is described. Section 6 presents the main results achieved by this study, i.e. the upper limits on annihilation cross section for different

\footnotetext{
${ }^{1}$ In the spherically symmetric model of $\mathrm{dSph}$, the DM density profile is a function of the halo-centric radius. A scale radius is representative of the extension of the innermost DM density profile. According to the kinematic data of member stars it sets a limit beyond of which the density profile is steeply falling and even the expected DM emission. Thus an obvious choice for a conservative truncation radius of DM annihilation emission is that of the outermost member star $\left(r_{\max }\right)$ used to estimate the velocity dispersion profile. Then here $\theta_{\max }$ is the angle corresponding to $r_{\max }$, i.e. the median estimated distance of the outermost member star from the center of the system.
} 
considered annihilation channels. Finally, the summary and conclusions of this work are given in Section 7.

\section{Expected gamma-ray flux from the DM halo of UMaII}

The gamma-ray flux ( $d \Phi / d E)$ produced by DM annihilation (or decay) arriving at Earth from a given region of the sky $(\Delta \Omega)$ is proportional to the product of two terms. The first one is the so-called particle physics factor:

$$
\frac{d \Phi^{\mathrm{PP}}}{d E}=\frac{1}{4 \pi} \frac{\left\langle\sigma_{\mathrm{ann}} v\right\rangle}{2 m_{\mathrm{DM}^{2}}} \sum_{i} B r_{i} \frac{d N_{\gamma}}{d E}
$$

This factor contains all the information relative to the specific DM particle model, $\left\langle\sigma_{\text {ann }} v\right\rangle$ is the thermal averaged annihilation cross section of the DM particle, $m_{\mathrm{DM}}$ is the mass of the DM particle, $B r_{i}$ is the branching ratio of the annihilation channel $i, N_{\gamma}$ is the number of gamma rays produced per annihilation reaction, and $E$ is the energy. The second term is the astrophysical (or $J$ ) factor, which accounts for the DM distribution and the distance of the source:

$$
J(\Delta \Omega)=\int_{\Delta \Omega} d \Omega^{\prime} \int_{\text {l.o.s. }} \rho^{2}\left(l, \Omega^{\prime}\right) d l \quad,
$$

where $\rho$ is the DM density profile. The integrals run over the line-of-sight (l.o.s.) and the observed sky region $(\Delta \Omega)$. Empirical estimates of DM content in dSphs, and hence the magnitudes of expected signals rely on inferences from stellar-kinematic data, through the Jeans equation (as closely treated in $[44,45]$ ). The wide literature on DM profile evaluation suggests that this topic requires different aspects to be evaluated when modeling galaxy DM distribution: kinematic and distribution of stars, estimated size of galaxy, in addition to the evaluation of specific stellar content, that accounts for baryons feedback. Therefore, DM profile parameterization, velocity anisotropy, and light profile modeling are needed to compute the $J$-factor and its uncertainties. Hence, the statistical uncertainties associated to the $J$-factors are due to finite sizes of stellar-kinematic data of member stars. Systematic uncertainties regard the shapes of DM density profiles as well as systematic errors can arise due to different stellar density profiles, non-spherical symmetry, and more complicated behaviors of the velocity anisotropy [46].

Annihilation of WIMPs could result in different types of gamma-ray signatures. First of all, a flux of gamma rays is expected from the $\pi^{0}$ decays resulting from the hadronization of SM particles produced in the DM annihilation/decay processes, and from the QCD and QED Final-State Radiation (FSR) [47]. The resulting gamma-ray spectra are continuous, with a cutoff at the kinematical limit (i.e. at the mass of the DM particle). Other processes producing sharp, monochromatic line are in most scenarios loop-suppressed and not considered here.

This work focuses on searching for the DM annihilation signal. We considered the DM annihilating into the SM pairs $b \bar{b}, W^{+} W^{-}, \tau^{+} \tau^{-}$, and $\mu^{+} \mu^{-}$, employing the average gammaray spectrum per annihilation process $\left(d N_{\gamma} / d E\right)$ computed for a set of DM particles of masses between $10 \mathrm{GeV}$ and $100 \mathrm{TeV}$ on the base of the PPPC 4 DM ID code realized on the PYTHIA simulation package version 8.135 [48].

Since the discovery of UMaII [40], several studies have been published on the $J$-factor estimate for this target [45, 49-53], all essentially confirming UMaII at the top of the ranking of highly promising dSph candidates for indirect DM searches. For our study, we use the 
UMaII $J$-factor parameterization as a function of the angular distance to the DM halo center given in [49], which is largely compatible with the other determinations found in literature. In particular, in our analysis we considered the value of $\log _{10}\left(J\left(\theta_{\max }\right)\left[\mathrm{GeV}^{2} \mathrm{~cm}^{-5}\right]\right)=19.42_{-0.42}^{+0.44}$ for the astrophysical factor integrated up to the maximum radius of the UMaII DM halo.

\section{MAGIC and the UMaII observation campaign}

The MAGIC (Major Atmospheric Gamma Imaging Cherenkov) telescopes are a system of two $17 \mathrm{~m}$ diameter telescopes located at the Roque de los Muchachos Observatory $\left(28.8^{\circ} \mathrm{N}\right.$, $17.9^{\circ} \mathrm{W} ; 2200 \mathrm{~m}$ a.s.l.), in the Canary island of La Palma (Spain). The two telescopes are both equipped with a fast imaging camera of $3.5^{\circ}$ field of view and are able to detect cosmic gamma rays in the VHE domain through the Cherenkov light produced by the atmospheric showers initiated by cosmic particles entering the Earth atmosphere. The whole MAGIC system underwent several hardware upgrades [54] and since its latest upgrade (accomplished in summer 2014 [55]) the system has improved considerably its performance close to the energy threshold, currently providing the world-best sensitivity around $\sim 100 \mathrm{GeV}$. This represents a crucial achievement for indirect DM searches at VHE, given the typical continuum spectra expected from DM annihilation/decay processes (which makes a good sensitivity at low energy threshold a key performance factor).

UMaII was observed by MAGIC between December 2014 and April 2016, for a total of 106.8 hours. Since the observations started right after the latest upgrade of the system, the whole data sample was taken with the same hardware conditions and optimal performance. The survey was carried out in the false source tracking (or "wobble") mode [56], in which two wobble positions offset by $0.4^{\circ}$ from the center of target in opposite RA direction were alternated every 20 minutes. For each wobble pointing direction, the residual background associated to the $\mathrm{ON}$ region around UMaII was estimated from the (OFF) region placed at the same relative location with respect to the pointing direction of the complementary wobble observation. With this configuration, the distance between the center of the $\mathrm{ON}$ and $\mathrm{OFF}$ nominal positions in the MAGIC cameras was always kept at $0.8^{\circ}$. The data were taken at medium zenith angles, ranging between $\sim 35^{\circ}$ and $\sim 45^{\circ}$, being the culmination of the source at MAGIC site at $35^{\circ}$. This resulted in an analysis energy threshold (defined as the peak of the energy distribution for a Monte Carlo simulated Crab-Nebula like gamma-ray data set after all analysis cuts) of $\sim 120 \mathrm{GeV}$.

\section{Standard data reduction and results}

The standard data reduction was performed with MARS [57], the official MAGIC data reconstruction and analysis package. Data quality selection was based on LIDAR information [58]. The selection resulted in 94.8 hours of excellent-quality data. After the standard data calibration, image cleaning and parameterization, events with a total amount of signal in the recorded showers below 50 photo-electrons (for any telescope) were rejected. Then, the main stereo parameters were calculated combining the information coming from the individual telescopes. The gamma/hadron separation was achieved by means of a multivariate method called Random Forest (RF) [59]. The algorithm employs basic image, timing and stereo parameters to compute a gamma/hadron discriminator called Hadronness by comparison of real (hadronicdominated) data with dedicated Monte Carlo gamma-ray simulations. The estimate of the arrival direction of the events was performed as well with the RF method, making use solely 
of MC gamma-ray simulations. This quantity was eventually used to compute the so-called $\theta^{2}$ parameter, which is the squared angular distance between the reconstructed event direction and the nominal position of the target. Finally, the energy reconstruction of the events was achieved by averaging individual energy estimators for both telescopes based on look-up tables [60]. The whole standard analysis procedures was validated by means of contemporaneous Crab Nebula observations (at the same zenith range of UMaII observations), which provided the expected performance in terms of sensitivity and spectral behavior.

The analysis cuts were optimized by means of a dedicated procedure aimed at finding the best sensitivity ${ }^{2}$ to the thermally averaged annihilation cross section $\left\langle\sigma_{\text {ann }} v\right\rangle$ of the full likelihood analysis (see Sec. 5) as a function of different cuts in Hadronness and $\theta^{2}$ parameters. The extension of the source was taken into account in the optimization of the cuts as explained in Sec 5.

Defining the profile likelihood ratio $\lambda_{P}[61]$ as a function of $\langle\sigma v\rangle$ for the measured dataset $\mathcal{D}:$

$$
\lambda_{P}(\langle\sigma v\rangle \mid \mathcal{D})=\frac{\mathcal{L}(\langle\sigma v\rangle ; \hat{\hat{\nu}} \mid \mathcal{D})}{\mathcal{L}(\widehat{\langle\sigma v\rangle} ; \hat{\nu} \mid \mathcal{D})},
$$

where $\mathcal{L}$ is the likelihood function (whose detailed expression is the Eq. 5.1), depending on the nuisance parameters $\nu$ (i.e. the ratio of exposures between the OFF and ON regions and the expected number of background events in the OFF region, see next section), in particular $\hat{\nu}$ and $\widehat{\langle\sigma v\rangle}$ are the values maximizing it, and the $\hat{\hat{\nu}}$ maximizes $\mathcal{L}$ for a given value of $\langle\sigma v\rangle$. The sensitivity can be approximated by $\langle\sigma v\rangle_{\mathrm{svt}}=\langle\sigma v\rangle_{2.71}-\widehat{\langle\sigma v\rangle}$, where $\langle\sigma v\rangle_{2.71}$ is defined by $-2 \ln \lambda_{P}\left(\langle\sigma v\rangle_{2.71} \mid \mathcal{D}\right)=2.71$ (for a detailed explanation, see [20]). As result of the optimization cuts procedure, the optimal cuts $\theta=0.3^{\circ 3}$ and Hadronness retaining $70 \%$ MC gamma rays - independently in 40 logarithmic energy bin cuts between $10 \mathrm{GeV}$ and $100 \mathrm{TeV}$ - provided the best choice for the gamma/hadron separation cuts for all considered final states and two benchmark DM masses: $1 \mathrm{TeV}$ and $10 \mathrm{TeV}$ (i.e. where the most stringent constraints can be typically achieved).

The overall search for a gamma-ray signal from UMaII was performed with the so-called $\theta^{2}$-plot, after the application of the energy-dependent optimized cuts, and within the chosen integration $\theta^{2}$ region. In order to evaluate the residual background of the observation, the $\theta^{2}$ distribution around a nominal background control region was also calculated. Figure 1 shows the resulting $\theta^{2}$-plot. No significant gamma-ray excess ${ }^{4}$ was found around the nominal position of UMaII.

In Figure 2 the sky-map [63,64] centered in the target sky position calculated with the application of the same analysis cuts is depicted. Our test statistic is taken from [65] (Eq. 17), applied on a smoothed and modelled background estimation. The statistical hypothesis tested, that is the null hypothesis, mostly resembles a $\chi^{2}$ distribution. This circumstance allows to apply the Wilks theorem [66] in order to estimate the level of agreement between the data and the hypothesis, inferring the significance of the observed result. Also in this case, no significant gamma-ray excess over the background in the sky region of UMaII DM halo (yellow dashed circle) was found.

\footnotetext{
${ }^{2}$ Here, "sensitivity" is defined as the average upper limit that would be obtained by an ensemble of experiments with the expected background and no signal [62].

${ }^{3}$ Due to the extended estimated size of the UMaII DM halo $\left(\theta_{\max } \simeq 0.53^{\circ}\right)$, we evaluated that the leakage of the signal into the chosen OFF region of $0.3^{\circ}$ is less than a thousandth of the total signal and, thus, negligeble.

${ }^{4}$ The deficit found in the $\theta^{2}$-plot $(\simeq-2 \sigma)$ is at the level of $1 \%$ of the background, i.e. within the systematic effect of $1.5 \%$ properly taken into account in the likelihood analysis (see section 5 ).
} 


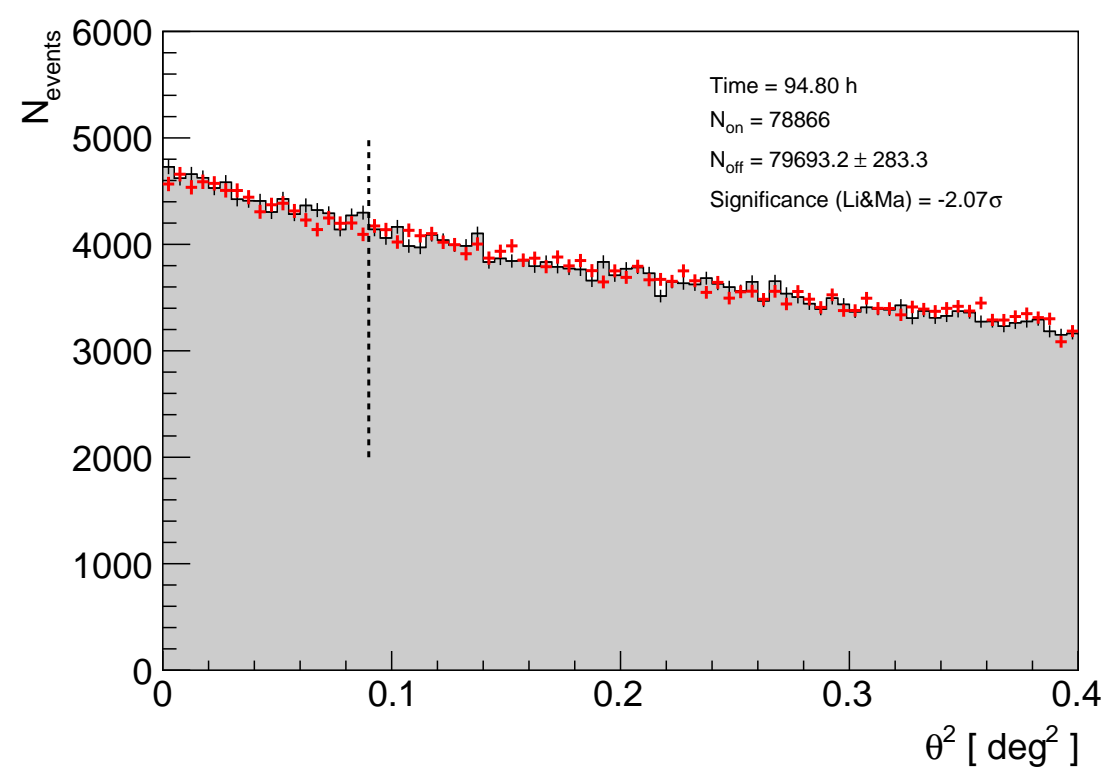

Figure 1: $\theta^{2}$ distributions of ON (red) and OFF (grey) regions resulting from 94.8 hours of MAGIC stereoscopic observations of UMaII taken between December 2014 and April 2016, with an energy threshold of $120 \mathrm{GeV}$. The region between zero and the vertical dashed line (at $\theta^{2}=0.09^{\circ 2}$ ) represents the $\mathrm{ON}$ and $\mathrm{OFF}$ integration regions.

Since no hint of a gamma-ray excess was found, the analysis of UMaII data proceeded with the computation of the constraints on the DM annihilation cross-section for different considered channels, using the full likelihood analysis method. In the next sections, before presenting the final results achieved in this work, we discuss the analysis method and its input quantities.

\section{$5 \quad$ Full likelihood analysis method}

The full likelihood allows the exploitation of spectral features of the expected DM signal to optimize the sensitivity with respect to a conventional analysis approach. A detailed review of this method and its formalism can be found in [67].

The likelihood $\mathcal{L}$ is a function depending on the expected number of gamma-rays $g$ detected as a function of the estimated energy $E^{\prime}$ and an observation time $T_{\text {obs. }}$. In addition, the likelihood depends on several nuisance parameters (for a detailed explanation of the likelihood function see [20]). In this study we performed a binned analysis, i.e. we considered $N_{\text {bins }}$ bins in estimated energy in the full likelihood function introduced in [67] (and used in the previous MAGIC DM studies [20,30]). Here $\mathcal{L}$ is the product of two likelihood functions $\mathcal{L}_{i}$, one for each set of data taken in the two different wobble pointing directions $(i)$. The 


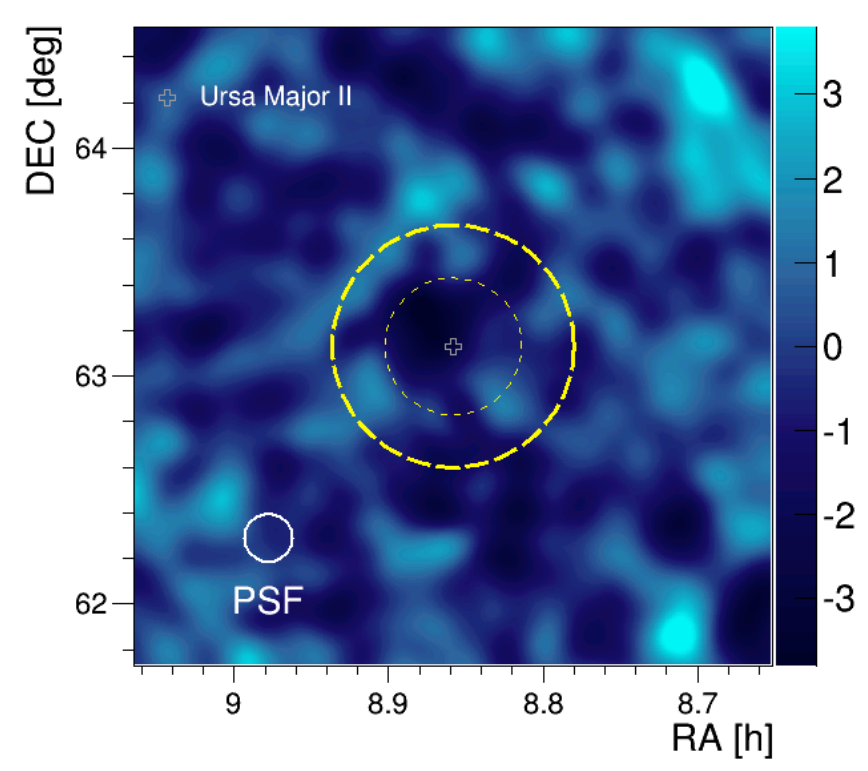

Figure 2: Significance sky-map centered at the UMaII sky position from 94.8 hours of MAGIC stereoscopic observations taken between December 2014 and April 2016, with an energy threshold of $120 \mathrm{GeV}$. The UMaII center position is marked with an empty white cross. The color scale represents the test statistic value distribution. The dashed yellow (external) circle represents the region within the maximum-radius of $0.53^{\circ}$ of the UMaII DM halo. The dotted yellow (internal) circle represents the region within the optimized analysis $\theta$ cut of $0.3^{\circ}$. The MAGIC PSF (for the given analysis cuts) of $0.11^{\circ}$ is also shown (white circle).

binned version reads as:

$$
\begin{aligned}
\mathcal{L}_{i}\left(\langle\sigma v\rangle ; \boldsymbol{\nu}_{i} \mid \mathcal{D}_{i}\right)= & \mathcal{L}_{i}\left(\langle\sigma v\rangle ;\left\{b_{i j}\right\}_{j=1, \ldots, N_{\mathrm{bins}}}, J, \tau_{i} \mid\left(N_{\mathrm{ON}, i j}, N_{\mathrm{OFF}, i j}\right)_{j=1, \ldots, N_{\mathrm{bins}}}\right) \\
= & \prod_{j=1}^{N_{\mathrm{bins}}}\left[\frac{\left(g_{i j}(\langle\sigma v\rangle)+b_{i j}\right)^{N_{\mathrm{ON}, i j}}}{N_{\mathrm{ON}, i j} !} e^{-\left(g_{i j}(\langle\sigma v\rangle)+b_{i j}\right)}\right. \\
& \left.\times \frac{\left(\tau_{i} b_{i j}\right)^{N_{\mathrm{OFF}, i j}}}{N_{\mathrm{OFF}, i j} !} e^{-\left(\tau_{i} b_{i j}\right)}\right] \\
& \times \mathcal{T}\left(\tau_{i} \mid \tau_{\mathrm{obs}, i}, \sigma_{\tau, i}\right) \times \mathcal{J}\left(J \mid J_{\mathrm{obs}}, \sigma_{\log _{10} J}\right)
\end{aligned}
$$

where the index $i=1,2$. The $\boldsymbol{\nu}_{i}$ represents the nuisance parameters and $\boldsymbol{D}_{i}$ the dataset; $g_{i j}$, $b_{i j}$ and $N_{\mathrm{ON}, i j}$ are the estimated number of signal and background events, and the number of observed events, respectively, in the $j$-th ON energy bin; $N_{\mathrm{OFF}, i j}$ is the number of observed events in the corresponding OFF bin; $\mathcal{J}$ is the likelihood for the $J$-factor, $\mathcal{T}$ is the likelihood for $\tau_{i}$ (the $\mathrm{OFF} / \mathrm{ON}$ acceptance ratio), determined from the ratio of the number of observed events in regions adjacent to the OFF and ON regions, parameterized by a Gaussian function with mean $\tau_{\text {obs }, i}$ and variance $\sigma_{\tau, i}^{2}$, which include statistical and systematics uncertainties. In the present analysis, we considered a systematic uncertainty of $\sigma_{\tau_{\text {syst }}}=1.5 \%$ on the estimate of the residual background (see the Table 1). This value has been established on the base of a dedicated performance study [55]. At high statistics $\left(>10^{4} \mathrm{ON}\right.$ events, corresponding to 


\begin{tabular}{|c|c|c|c|c|}
\hline Wobble position & $\begin{array}{c}\text { Eff ON time } \\
{[\mathrm{h}]}\end{array}$ & $\tau$ & $\sigma_{\tau_{\text {stat }}}$ & $\sigma_{\tau_{\text {syst }}}$ \\
\hline $\mathrm{W} 1$ & 49.29 & 0.9111 & 0.0037 & 0.0137 \\
\hline $\mathrm{W} 2$ & 45.49 & 1.0943 & 0.0045 & 0.0164 \\
\hline
\end{tabular}

Table 1: Effective observation time (second column), ON/OFF acceptance ratio $\tau$ (third column), statistic error for $\tau$ (fourth column), systematic error for $\tau$ (last column) considered in this analysis, for both wobble pointing positions (first column).

$\sim 50 \mathrm{~h}$ ), the systematic uncertainty dominates and is due to the possible difference in camera acceptance between the ON and OFF regions. $b_{i j}, J$ and $\tau_{i}$ are nuisance parameters, whereas $g_{i j}$ depend on the free parameter $\langle\sigma v\rangle$ through:

$$
g_{i j}(\langle\sigma v\rangle)=T_{\mathrm{obs}, i} \int_{E_{\min , j}^{\prime}}^{E_{\max , j}^{\prime}} d E^{\prime} \int_{0}^{\infty} d E \frac{d \Phi(\langle\sigma v\rangle)}{d E} A_{\mathrm{eff}}(E) G\left(E^{\prime} \mid E\right),
$$

where $T_{\mathrm{obs}, i}$ is the total observation time, $E$ and $E^{\prime}$ the true and estimated gamma-ray energy, respectively, and $E_{\min , j}^{\prime}$ and $E_{\max , j}^{\prime}$ the minimum and maximum energies, respectively, of the $j$-th energy bin. Finally, $A_{\text {eff }}$ is the effective collection area and $G$ the probability density function (PDF) of the energy estimator, both computed from a Monte Carlo simulated gamma-ray dataset following the spatial distribution expected for DM-induced signals from UMaII (see appendix A for further details).

The input of the likelihood are the number of events detected in the ON and OFF regions for the different bins in estimated energy -after proper cuts in Hadronness and $\theta^{2}$ parameteras well as the instrument response functions (IRFs) computed for the specific observation period and the extension of the source.

$\mathrm{UMaII}$ is an extended source for the MAGIC PSF $\left(\sim 0.1^{\circ}\right)$, being $\theta_{\max }=0.53^{\circ}$ and the "half-light-radius" equal to $\theta_{0.5}=0.24^{\circ}$. For this reason, in order to take into acount the extension of DM emission region, the IRFs were computed from MC simulations following UMaII morphology (see details in appendix A).

Using the profile likelihood ratio $\lambda_{P}$ (see Eq. 4.1) we test hypotheses that assume the flux computed with Eq. 2.1 and 2.2, considering "pure" annihilation channels: $b \bar{b}, \tau^{+} \tau^{-}, \mu^{+} \mu^{-}$, and $W^{+} W^{-}$; one-sided $95 \%$ confidence level (CL) limits are given by the largest of the two $\langle\sigma v\rangle_{2.71}$ solutions (as defined in section 4).

\section{Results on dark matter annihilation models}

In this section we present the 95\% CL upper limits on the thermally-averaged cross-section $\left\langle\sigma_{\mathrm{ann}} v\right\rangle$ for DM particles annihilating with $100 \%$ branching ratio into different SM particle pairs achieved in 94.8 hours of selected data of the UMaII campaign. The search was performed for DM particles of masses between $100 \mathrm{GeV}$ and $100 \mathrm{TeV}$ for annihilation scenarios. In our full likelihood approach, we followed the same prescription adopted in [20], restricting the value of $\left\langle\sigma_{a n n} v\right\rangle$ to the physical $(\geq 0)$ region. Furthermore, no additional boosts, either from the presence of substructures [68] or from quantum effects [69], were assumed for computing the final results.

In Figure 3, the 95\% CL upper limits on $\left\langle\sigma_{\text {ann }} v\right\rangle$, for DM particles annihilating into $b \bar{b}$, $W^{+} W^{-}, \tau^{+} \tau^{-}$, and $\mu^{+} \mu^{-}$, achieved after the application of the optimized cuts and with a 
binned $\left(N_{\text {bins }}=30\right)$ likelihood analysis are shown. In addition, the two-sided $68 \%$ and $95 \%$ containment bands for the distribution of limits under the null hypothesis are also reported. The containment bands were computed from the distribution of the upper limits obtained from the analysis of 1000 realizations of the null hypothesis $(\langle\sigma v\rangle=0)$, consisting of fast simulations (for both ON and background regions) generated from background PDFs, assuming similar exposures as for the real data, and $J$-factors assumed as nuisance parameters in the full likelihood function. All bounds are consistent with the no-detection scenario. The achieved
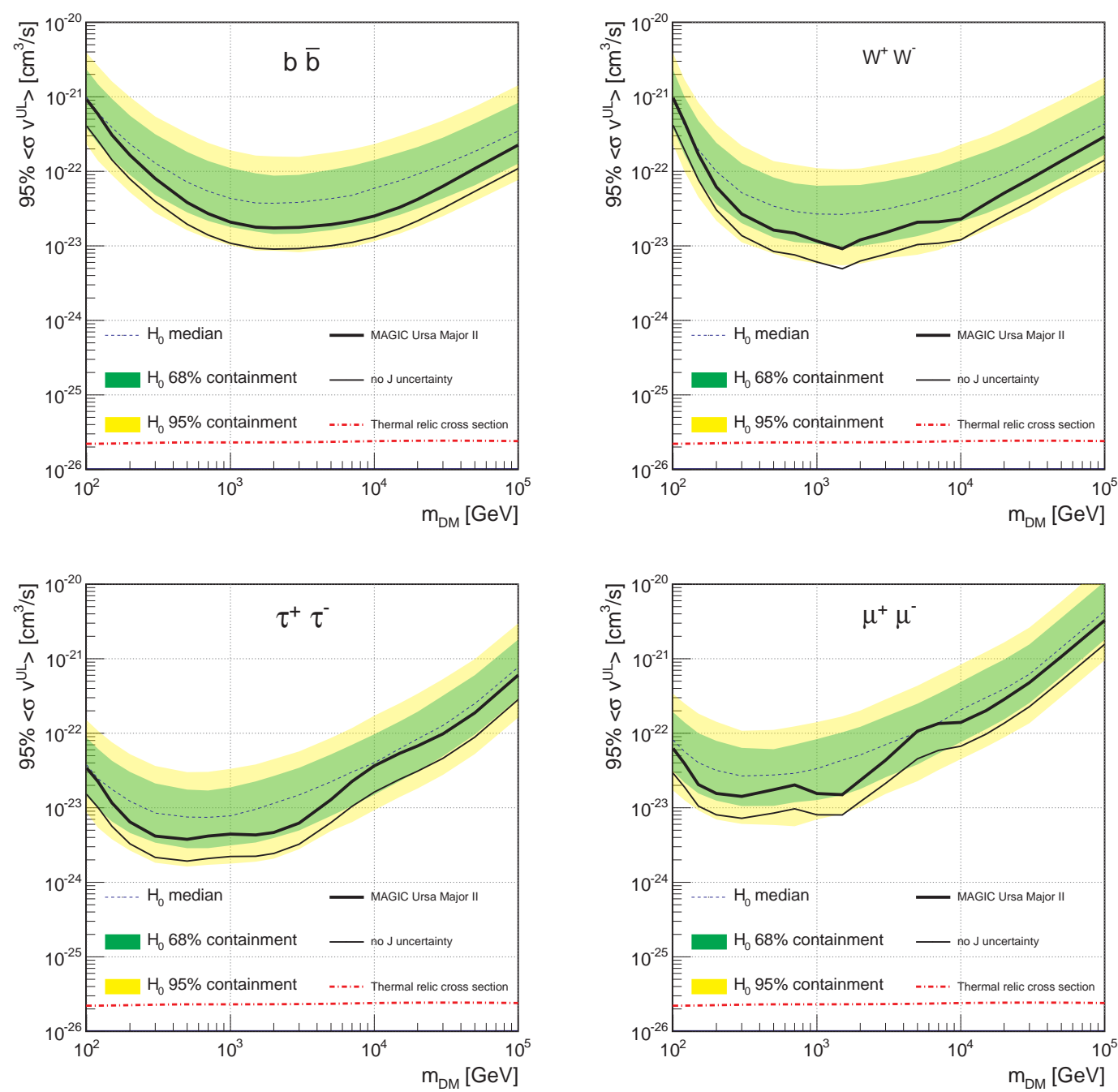

Figure 3: 95\% CL upper limits on $\left\langle\sigma_{\mathrm{ann}} v\right\rangle$ for DM particles annihilating into $b \bar{b}$ (upperleft), $W^{+} W^{-}$(upper-right), $\tau^{+} \tau^{-}$(bottom-left) and $\mu^{+} \mu^{-}$(bottom-right) pairs. Thick-solid and thin-solid lines show, respectively, the limits obtained with $94.8 \mathrm{~h}$ of UMaII observation, considering the $J$-factor a nuisance parameter and fixing its value in the likelihood. The thindotted line, green and yellow bands show, respectively, the median and the symmetrical, twosided $68 \%$ and $95 \%$ containment bands for the distribution of limits under the null hypothesis. The red-dashed-dotted line shows the thermal relic cross-section from [9]. 
results represent among the most stringent and robust constraints to the annihilation crosssection obtained from observations of single $\mathrm{dSphs}$, in the $\mathrm{TeV}$ mass region. In particular, our strongest limit $(95 \% \mathrm{CL})$ corresponds to a $\sim 0.5 \mathrm{TeV}$ DM particle annihilating into $\tau^{+} \tau^{-}$, and is of order $\left\langle\sigma_{\text {ann }} v\right\rangle \simeq 3.8 \times 10^{-24} \mathrm{~cm}^{3} \mathrm{~s}^{-1}$. The results are comparable with the recently published limits achieved by VERITAS Collaboration in the joint analysis of data collected on four dSphs (for a total of 216 hours of collected data) [33] and the combined results of HESS campaigns on dSphs (including 5 dSphs for an amount of 140 hours) [28].

Due to the difference in the analysis method developed and adopted for the UMaII data with respect to the previous dSph campaigns, mainly in the treatment of the nuisance parameter $J$ and in the background modeling, a straightforward comparison with previous MAGIC results is not easily achievable. Nevertheless, the results shown in the present work are comparable (within a factor of $\sim 3$ ) with those obtained with the Segue 1 campaign ( $\sim 160$ hours) [30], once the difference in the targets' exposure, the treatment of the systematics for $\tau$, and other differences in the analyses are taken into account. We reserve for future publications to combine all MAGIC data collected on dSphs in a new homogeneous analysis, that will take advantage of the optimized tools tested and used in the present work.

\section{Summary and Conclusions}

The MAGIC telescopes conducted a deep observation campaign at VHE toward the UMaII $\mathrm{dSph}$, a promising target for indirect DM searches. The source was observed between December 2014 and April 2016, resulting in 94.8 hours of excellent-quality data. This campaign represents an important step toward an optimal "target diversification strategy" at VHE aimed at enhancing the chances of discovery of DM signals and reducing possible biases in target selection.

Since no significant gamma-ray excess was found in the UMaII data, the observations were used to derive the constraints to the annihilation cross-section assuming annihilation into the SM pairs $b \bar{b}, W^{+} W^{-}, \tau^{+} \tau^{-}$, and $\mu^{+} \mu^{-}$, for DM particles in the $100 \mathrm{GeV}-100 \mathrm{TeV}$ mass range. The $95 \%$ CL limits obtained in this work, by means of the full likelihood analysis method, are among the most stringent and robust achieved so far from observations of dSphs at the $\mathrm{TeV}$ mass scale. For the first time we optimized the DM search in dSph exploiting the morphology information of the target by taking into account the extension of the UMaII DM halo (see appendix A).

Since the beginning of the UMaII campaign with MAGIC, in the last two years new interesting dSphs for DM searches have been discovered. In this respect the MAGIC program of DM search in dSphs continues, following the target diversification strategy proposed with the UMaII campaign. Moreover, thanks to the full likelihood analysis method, the results of this work will have a natural development in a more general framework of joint analysis involving different $\mathrm{dSphs}$ and (possibly) different instruments.

\section{Acknowledgments}

We would like to thank the Instituto de Astrofísica de Canarias for the excellent working conditions at the Observatorio del Roque de los Muchachos in La Palma. The financial support of the German BMBF and MPG, the Italian INFN and INAF, the Swiss National Fund SNF, the ERDF under the Spanish MINECO (FPA2015-69818-P, FPA2012-36668, FPA2015-68378-P, 
FPA2015-69210-C6-2-R, FPA2015-69210-C6-4-R, FPA2015-69210-C6-6-R, AYA2015-71042P, AYA2016-76012-C3-1-P, ESP2015-71662-C2-2-P, CSD2009-00064), and the Japanese JSPS and MEXT is gratefully acknowledged. This work was also supported by the Spanish Centro de Excelencia "Severo Ochoa" SEV-2012-0234 and SEV-2015-0548, and Unidad de Excelencia "María de Maeztu" MDM-2014-0369, by the Croatian Science Foundation (HrZZ) Project IP-2016-06-9782 and the University of Rijeka Project 13.12.1.3.02, by the DFG Collaborative Research Centers SFB823/C4 and SFB876/C3, the Polish National Research Centre grant UMO-2016/22/M/ST9/00382 and by the Brazilian MCTIC, CNPq and FAPERJ. The work of the author M. Vazquez Acosta is financed with grant RYC-2013-14660 of MINECO.

\section{References}

[1] Einasto, J. (2014), Dark Matter and Cosmic Web Story, Edited by Jaan Einasto. Published by World Scientific Publishing Co. Pte. Ltd., 2013.(World Scientific Publishing Co)

[2] Rubin V. C. and Ford, W. K. Jr., Rotation of the Andromeda Nebula from a Spectroscopic Survey of Emission Regions, Astrophys. J. 159 (1970) 379-403.

[3] Rubin V. C., et al., Rotational properties of $21 \mathrm{SC}$ galaxies with a large range of luminosities and radii, from NGC $4605(\mathrm{R}=4 \mathrm{kpc})$ to UGC $2885(\mathrm{R}=122 \mathrm{kpc})$, Astrophys. J. 238 (1980) 471.

[4] Zwicky F., Spectral displacement of extra galactic nebulae, Helv. Phys. Acta 6 (1933) 110-127.

[5] Squires G. et al., The Dark Matter, gas and galaxy distributions in Abell 2218: a weak gravitational lensing and X-ray analysis, Astrophys.J. 461 (1996) 572, [arXiv:astro-ph/9507008].

[6] Bradač M. et al., Strong and weak lensing united III: Measuring the mass distribution of the merging galaxy cluster 1E0657-56, Astrophys. J. 652 (2006) pp. 937-947, [arXiv:astro-ph/0608408].

[7] Plank Collaboration, Ade P.A.R. et al., Planck 2015 results, XIII. Cosmological parameters, [arXiv:1502.0158].

[8] Feng J. L., Sep. 2010. Dark Matter Candidates from Particle Physics and Methods of Detection, ARA\&A 48, 495-545, [arXiv:1003.0904].

[9] Steigman G., Dasgupta B, and Beacom J. F., Precise relic WIMP abundance and its impact on searches for dark matter annihilation, Phys.Rev. D86(2012) 023506, [arXiv:1204.3622].

[10] Jungman G, M., et al., Supersymmetric dark matter, Phys.Rept. 267 (1996) 195-373, [arXiv:hep-ph/9506380].

[11] Salati P., Dark matter annihilation in the Universe, International Journal of Modern Physics: Conference Series c World Scientific Publishing Company, [arXiv:1403.4495v1].

[12] Balducci D., et al., Uncovering Natural Supersymmetry via the interplay between the LHC and Direct Dark Matter Detection, JHEP 1507 (2015) 066, [arXiv:1504.02472].

[13] Atlas Collaboration, Dark matter interpretations of ATLAS searches for the electroweak production of supersymmetric particles in $\sqrt[2]{s}$ TeV proton-proton collisions, JHEP09 (2016) 175, [arXiv:1608.00872]

[14] XENON100 Collaboration, Aprile E. et al., Dark Matter Results from 225 Live Days of XENON100 Data, Phys. Rev. Lett. 109, 181301 (2012), [arXiv:1207.5988].

[15] LUX Collaboration, Akerib D. S. et al., First results from the LUX dark matter experiment at the Sanford Underground Research Facility, Phys. Rev. Lett. 112, 091303 (2014), [arXiv:1310.8214]. 
[16] Agnes P., et al., First Results from the DarkSide-50 Dark Matter Experiment at Laboratori Nazionali del Gran Sasso, Physics Letters B 743, 456 (2015), [arXiv:1410.0653].

[17] Feng J., et al., Antiproton-to-proton Flux Ratio and Positron Flux Measured by AMS-02, [arXiv:1701.02263].

[18] Gaskins J. M., A review of indirect searches for particle dark matter, Contemporary Physics Vol. 57 , Iss. 4,2016, [arXiv:1604.00014].

[19] Naurois M., Mazin D., Ground-based detectors in very-high-energy gamma-ray astronomy, C.R. Physique 16 (2015) 610-627, [arXiv:1511.00463].

[20] Ahnen M. L., et al., Limits to dark matter annihilation cross-section from a combined analysis of MAGIC and Fermi-LAT observations of dwarf satellite galaxies, JCAP 02 (2016) 039, [arXiv:1601.06590].

[21] Livio M.\& Silk J., Physics: Broaden the search for dark matter, Nature 507, 29-31 (2014), [arXiv:1404.2591]

[22] Van Eldik C., Gamma rays from the Galactic Centre Region: a review, [arXiv:1505.06055].

[23] Cirelli M, et al., Gamma ray constraints on decaying Dark Matter, Phys.Rev. D86 (2012) 083506, [arXiv:1205.5283].

[24] Martinez G. D., et al., Indirect Dark Matter detection from dwarf Satellites: joint expectations from Astrophysics and Supersymmetry, JCAP 0906 (2009) 014, [arXiv:0902.4715].

[25] Persic M., The Universal Rotation Curve of Spiral Galaxies: I. the Dark Matter Connection, MNRAS 281 (1996) 27-47 (and Erratum: MNRAS, 283, 1102), [arXiv:astro-ph/9506004].

[26] Doro M., A decade of dark matter searches with ground-based Cherenkov telescopes, Nuclear Instruments and Methods in Physics Research Section A, 742 (2014) 99-106, [arXiv:1404.5017].

[27] H.E.S.S. Collaboration, Abdallah H., Search for dark matter annihilations towards the inner Galactic halo from 10 years of observations with H.E.S.S, Phys. Rev. Lett. 117, 111301 (2016), [arXiv:1607.08142].

[28] HESS Collaboration, Abramowski A., et al., Search for dark matter annihilation signatures in H.E.S.S. observations of Dwarf Spheroidal Galaxies, Phys. Rev. D 90, 112012 (2014), [arXiv:1410.2589].

[29] Aleksić J. et al., Searches for Dark Matter annihilation signatures in the Segue 1 satellite galaxy with the MAGIC-I telescope, JCAP 1106:035,2011, [arXiv:1103.0477].

[30] Aleksić J. et al., Optimized dark matter searches in deep observations of Segue 1 with MAGIC, JCAP 02 (2014) 008, [arXiv:1312.1535].

[31] Doro M., A review of the past and present MAGIC dark matter search program and a glimpse at the future, XXV ECRS 2016 Proceedings - eConf C16-09-04.3, [arXiv:1701.05702].

[32] Zitzer B. for the VERITAS CollaborationThe VERITAS Dark Matter Program, 2014 Fermi Symposium proceedings - eConf C14102.1, [arXiv:1503.00743]

[33] VERITAS Collaboration, Archambault S., et al., Dark Matter Constraints from a Joint Analysis of Dwarf Spheroidal Galaxy Observations with VERITAS, Physical Review D 95, 082001 (2017), [arXiv:1703.04937].

[34] Battaglia G., Helmi A., Breddels M., Internal kinematics and dynamical models of dwarf spheroidal galaxies around the Milky Way, New Astronomy Review, Vol. 57, Issues 3-4, Pages 59-72, [arXiv:1305.5965].

[35] Ullio P. et al., A critical reassessment of particle Dark Matter limits from dwarf satellites, JCAP 07 (2016) 025, [arXiv:1603.07721]. 
[36] Fukugita M., et al., The Sloan Digital Sky Survey Photometric System, Astronomical Journal v.111, p.1748.

[37] DES Collaboration, Abbott T., et al. ,The Dark Energy Survey: more than dark energy - an overview, MNRAS 460 (2016) no. 2, 1270-1299, [arXiv:1601.00329].

[38] Chambers K. C., et al., The PAN-STARRS1 surveys, [arXiv:1612.05560].

[39] Antoja T., et al., Detection of satellite remnants in the Galactic Halo with Gaia- III. Detection limits for Ultra Faint Dwarf Galaxies, MNRAS (2015) 453 (1): 541-560, [arXiv:1507.04353].

[40] Zucker D. B., Belokurov V. et al., A curious new Milky Way satellite in Ursa Major, Astrophys.J.650: L41-L44,2006, [arXiv:astro-ph/0606633].

[41] Wolf J., et al., Accurate masses for dispersion-supported galaxies, 2010, MNRAS, 406,1220, [arXiv:0908.2995].

[42] Simon J. D. and Geha M., The kinematics of the ultra-faint Milky Way satellites: solving the missing satellite problem, Astrophys.J.670:313-331,2007, [arXiv:0706.0516].

[43] Giammaria P., Lombardi S. et al., Latest results on searches for Dark Matter signatures in galactic and extragalactic selected targets by the MAGIC Telescope, 2016, J. Phys. Conf. Ser., $718,042024$.

[44] Charbonnier A., Combet C. et al. Dark matter profiles and annihilation in dwarf spheroidal galaxies: prospectives for present and future Îş-ray observatories I. The classical dSphs, Mon. Not. R. Astron. Soc., 418, 1526 (2011), [arXiv1104:0412].

[45] Martinez M., A robust determination of Milky Way satellite properties using hierarchical mass modelling, Mon. Not. R. Astron. Soc. 451, 2524âĂŞ2535 (2015), [arxiv: 1309:2641].

[46] Bonnivard V., Combet C., Maurin D., and Walker M. G., Spherical Jeans analysis for dark matter indirect detection in dwarf spheroidal galaxies - Impact of physical parameters and triaxiality, 2015, MNRAS, 446, 3002, [arXiv:1407.7822].

[47] Cembranos J. A. R., et al., Reliability of Monte Carlo event generators for gamma-ray dark matter searches, JHEP 1309 (2013) 077, [arXiv:1305.2124].

[48] Cirelli M., et al., PPPC 4 DM ID: A Poor Particle Physicist Cookbook for Dark Matter Indirect Detection, JCAP 1103 (2011) 051, [arXiv:1012.4515].

[49] Geringer-Sameth A., Koushiappas S. M., Walker M., (2015), Dwarf galaxy annihilation and decay emission profiles for dark matter experiments, 2015 ApJ 801 74, [arXiv:1408.0002].

[50] Bonnivard V. et al. , Dark matter annihilation and decay in dwarf spheroidal galaxies: The classical and ultrafaint dSphs, Mon. Not. Roy. Astron. Soc. 453 (2015) 849-867, [arXiv:1504.02048].

[51] Hayashi K. et al., Dark matter annihilation and decay from non-spherical dark halos in the Galactic dwarf satellites, [arXiv:1603.08046].

[52] Sanders J. L. et al, Indirect Dark Matter Detection for Flattened Dwarf Galaxies, [arXiv:1604.05493].

[53] Chiappo A. et al., Dwarf spheroidal $J$-factors without priors: A likelihood-based analysis for indirect dark matter searches, [arXiv:1608.07111].

[54] Aleksić J., et al., The major upgrade of the MAGIC telescopes, Part I: The hardware improvements and the commissioning of the system, Astroparticle Physics Vol 72., [arXiv:1409.6073].

[55] Aleksić J., et al. (2016), The major upgrade of the MAGIC telescopes, Part II: A performance study using observations of the Crab Nebula, Astropart. Phys. 72 76-94, [arXiv:1409.5594]. 
[56] Formin V. P., et al., New method for atmospheric Cherenkov imaging for gamma-ray astronomy 1: The false source method, Astropart. Phys. 2 (1994) 137-150.

[57] Zanin R. et al., MARS, the MAGIC Analisis and Reconstruction Software, Proc. of the 33rd International Cosmic Ray Conference, Rio de Janeiro, Brasil, Braz.J.Phys. 44 (2014) no.5, pp.415-608.

[58] Fruck C., Gaug M., et al., A novel LIDAR-based Atmospheric Calibration Method for Improving the Data Analysis of MAGIC. In Proceedings of the 33rd ICRC, Rio de Janeiro, 2013, [arXiv:1403.3591].

[59] Albert J. et al., Implementation of the Random Forest Method for the Imaging Atmospheric Cherenkov Telescope MAGIC, Nucl. Instrum. Meth. A588 (2008) 424-432, [arXiv:0709.3719].

[60] Aleksić J., et al., 2012. Performance of the MAGIC stereo system obtained with Crab Nebula data. Astroparticle Physics, 35, 435-448, [arXiv:1108.1477].

[61] Olive K. A. et al. The Review of Particle Physics, Chin. Phys. C38 (2014) 090001.

[62] Feldman G. J. and Cousins R. D., A Unified Approach to the Classical Statistical Analysis of Small Signals, Phys. Rev. D57 (1998) 3873, [arXiv:physics/9711021]

[63] Aleksić J., et al., Observations of the Blazar 3C 66A with the MAGIC Telescopes in Stereoscopic Mode, 2011 ApJ, 726, 58, [arXiv:1010.0550]

[64] Lombardi S. et al., Advanced stereoscopic gamma-ray shower analysis with the MAGIC telescopes, Proc. of the 32nd International Cosmic Ray Conference, Beijing, China, (2011), [arXiv:1109.6195].

[65] Li T. P. and Ma Y. Q., Analysis methods for results in gamma-ray astronomy, ApJ, 272 (1983).

[66] Wilks S. The Large-Sample Distribution of the Likelihood Ratio for Testing Composite Hypotheses, Ann. Math. Statist. Vol 9, Number 1 (1938).

[67] Aleksić J., J. Rico and M. Martinez, Optimized analysis method for indirect dark matter searches with imaging air Cherenkov Telescopes, JCAP 10 (2012) 032, [arXiv:1209.5589].

[68] Strigari L., Precise constraints on the dark matter content of Milky Way dwarf galaxies for gamma-ray experiments, 2007, Phys. Rev. D, 75, 083526, [arXiv:astro-ph/0611925].

[69] Hisano J., et al., Non-perturbative effect on Dark Matter annihilation and Gamma Ray Signature from Galactic Center, Phys. Rev. D71 (2005) 063528, [arXiv:hep-ph/0412403].

[70] Ackerman M., et al., Search for extended sources in the Galactic Plane using 6 years of FermiLarge Area Telescope Pass 8 data above $10 \mathrm{GeV}$, [arXiv:1702.00476].

[71] Ahnen M. L., et al., Observations of Sagittarius A during the pericenter passage of the G2 object with MAGIC, Astro.Astrophys. Vol 601, 2017, [arXiv:1611.07095]

[72] Flinders A., VERITAS Observations of the Geminga Supernova Remnant, Proceedings, 34th International Cosmic Ray Conference(ICRC2015), The Hague, The Netherlands, [arXiv:1509.04224]

[73] Aliu E. et al., Spatially Resolving the Very High Energy emission from MGRO J2019+37 with VERITAS, Astrophys. J. 788, 2014, [arXiv1404.1841].

[74] Palacio J., Doro M., et al., Constraining the Dark Matter decay lifetime with very deep observations of the Perseus cluster with the MAGIC telescopes, Proceedings, 34th International Cosmic Ray Conference(ICRC2015), The Hague, The Netherlands, [arXiv:1509.03974]. 


\section{A DONUT Monte Carlo method}

Instrument response functions (IRFs) of Cherenkov telescopes are usually evaluated by means of Monte Carlo (MC) simulations. For many practical purposes, it is enough to evaluate IRFs for point-like gamma-ray sources. However, IRFs depend in general on the relative arrival direction of the gamma ray with respect to the telescope pointing direction. This means that the evaluation of IRFs for extended sources of arbitrary shape would in principle need a simulation of a gamma-ray sample with arrival directions distributed following the particular source morphology. Such morphology is expected to be very different from source to source (e.g.: the diffuse emission of the Milky Way plane [70, 71], nearby supernova remnants $[72,73]$ or the expected gamma emission from dark matter halos [30, 74]). In order to compute the IRFs applicable to the study of these sources, while making an efficient use of the computing resources devoted to MC simulations, we have developed a method, which we dub donut MC, described and characterized in this appendix.

MAGIC observations of point-like sources are carried out in wobble mode, i.e. with the telescope pointing successively at two or more directions $0.4^{\circ}$ away from the source position. The corresponding IRFs are computed using the so-called point-like $M C$, which consists of gamma rays simulated with true directions uniformly distributed in a ring centered at the telescope pointing direction and a radius of $0.4^{\circ}$ (see Figure 4, left) to cover all possible orientations between the pointing direction and the source position. Although extended sources, on the other hand, do not have a well defined source position, the wobbling procedure is still applied by pointing the telescope $0.4^{\circ}$ away from a certain direction that we call the source center. For evaluating the IRFs in this case, the natural procedure would be to simulate gamma rays with true directions following the source morphology around the source center, and the source centers uniformly distributed in a ring centered at the telescope pointing direction and a radius of $0.4^{\circ}$. Such dedicated MC production would demand at least as much computer resources as the point-like production, but would only be aplicable for the study of a very specific source morphology. As an effective alternative, we have developed a method to select simulated events from a MC production consisting of gamma rays with true directions uniformly distributed in a $1.5^{\circ}$ radius FoV (called diffuse $M C$, see Figure 4 , right). This procedure only adds a negligible overhead to the computing-intensive process of the full diffuse $\mathrm{MC}$ production, which is common to all possible source morphologies, thus making an efficient use of the computing resources available to MC simulations. For the case of a moderatelyextended, radially symmetric source, the distribution of true gamma-ray directions resulting from our procedure has the shape resembling that of a donut (see Figure 5, right), where the name of the method comes from. The rest of this appendix briefly describes the procedure of donut $\mathrm{MC}$ selection and of the consistency tests that show that our implementation actually produces the expected results. The donut MC method is the procedure by which we produce a MC sample, specific for the study of given source morphology, by selecting events from the diffuse MC (see Figure 4 right and Figure 5 right). The method maximizes the number of selected events in the new MC sample, while keeping them statistically uncorrelated.

In order to understand the procedure, let us first consider a simplified version, here named halo1, where we select events from the diffuse MC based on the source morphology for one single, fixed, orientation between the pointing direction and the source center (see Figure 5, left). If we used the halo1 sample to compute the IRFs corresponding to the assumed source morphology, we would get the correct result, but with large statistical uncertainties, 

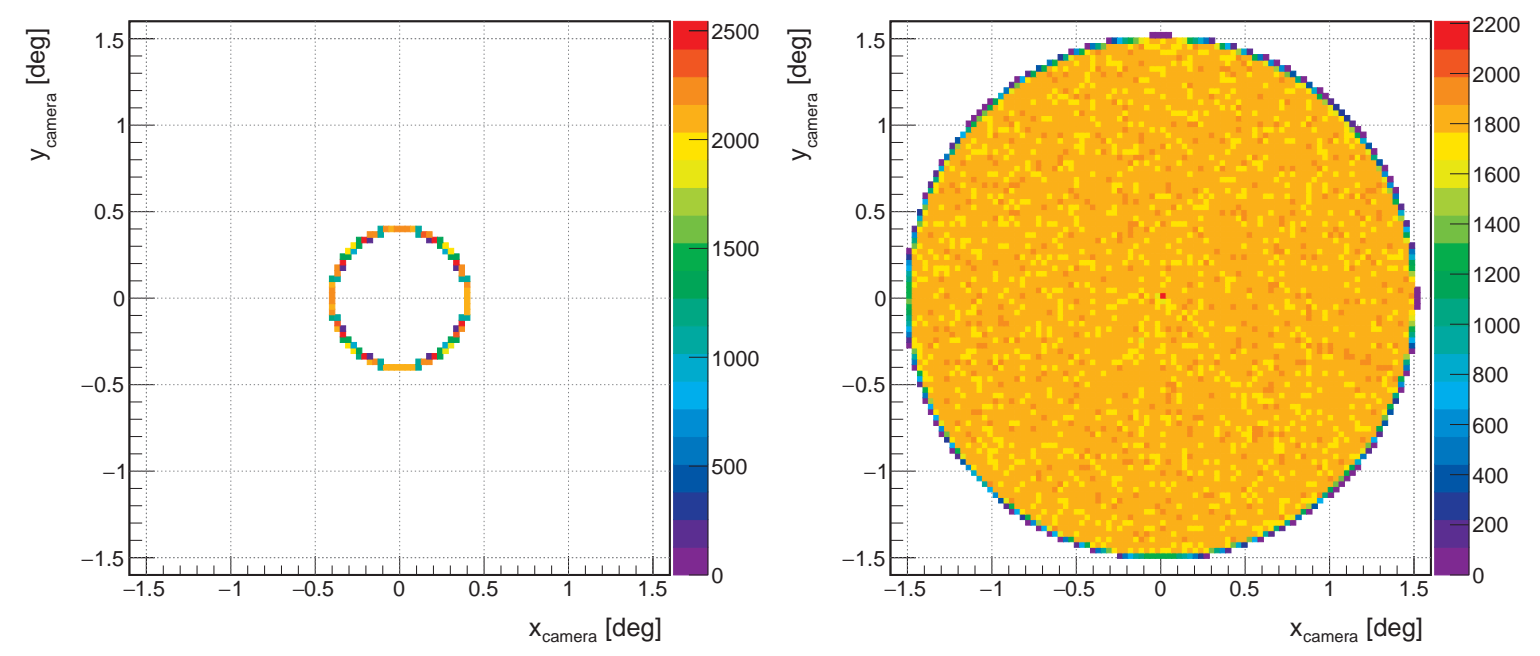

Figure 4: Distribution of true directions of simulated events in the point like (left) and diffuse (right) MAGIC MC productions, shown in camera coordinates.
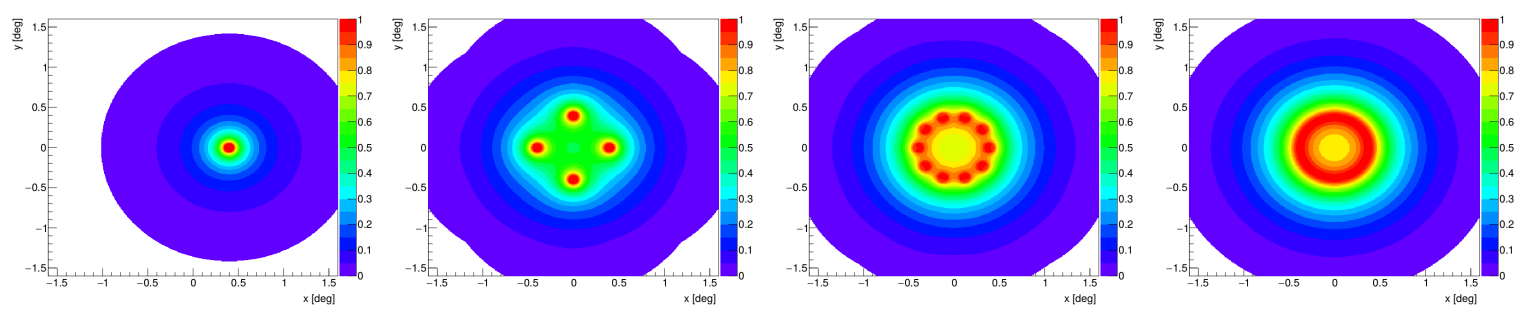

Figure 5: Expected distribution of gamma-ray true directions for different number of source center/pointing direction orientations (from left to right: halo1, halo4, halo10 and halo100) realizations shown in camera coordinates, for a given typical radially symmetric source. Image in the most right corresponds to the donut method joint PDF (see text for further explanations).

given the relatively low statistics of the selected sample with respect to the original one, and only valid for one possible orientation between pointing direction and source center. We can generalize the halo1 selection procedure for $n$ halos (halo4, halo10 and halo100 cases are shown in the right-most plots of Figure 5). If halo-n were constructed simply by repeating the selection procedure of halo1, the probability of having an event selected more than once, will get larger, the larger the value of $n$ becomes. In the donut method, this problem is solved by selecting diffuse MC events according to a joint probability density function from the convolution of all possible source center/pointing direction orientations (see Figure 5, most right). Selected events are associated with a source center randomly chosen from the expected $0.4^{\circ}$ ring such that, at the end of the selection process, all events with a common source center are spatiallly distributed according to the source morphology. To show that this procedure works as expected we have performed the following tests:

- Check that the distributions of event directions with respect to the pointing direction (see Figure 6 left) and the associated source center (see Figure 6 right) agree, within 

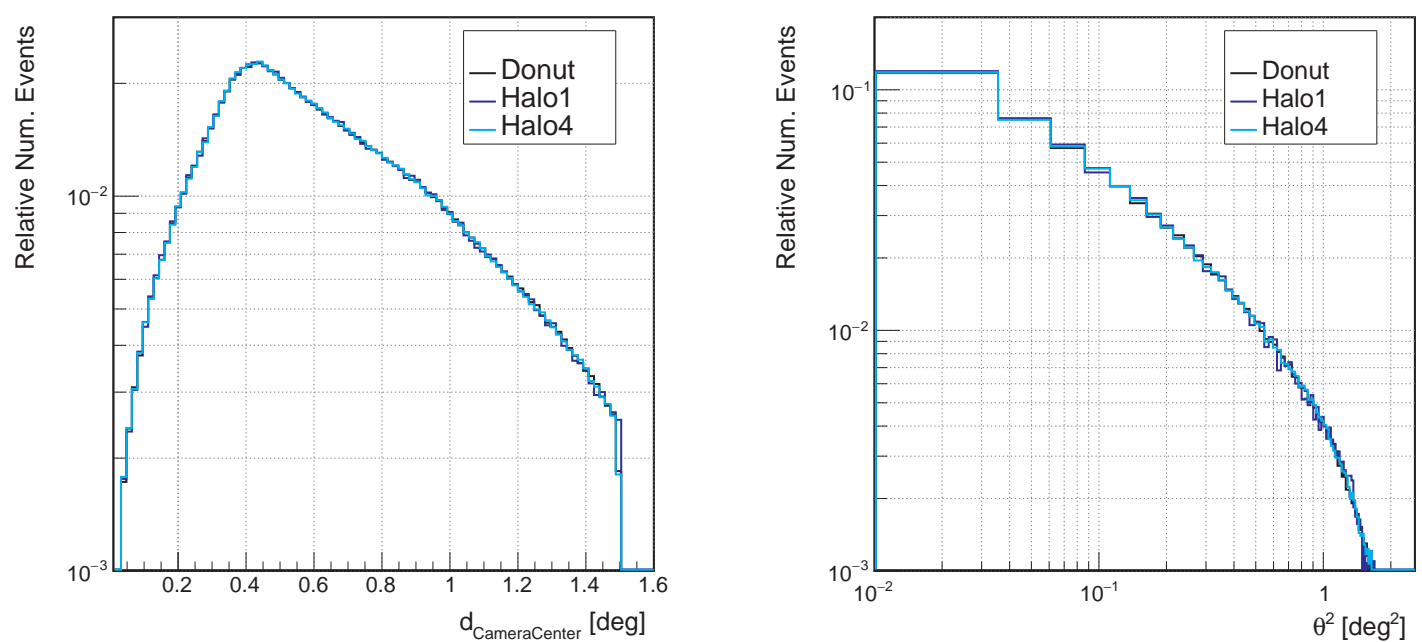

Figure 6: Comparison between halo1 (red), halo4 (pink) and donut MC (green). The left plot shows the distribution of events as a function of the distance to the pointing direction. The right plot shows the distribution of distances squared to the associated source center.

statistical uncertainy, for the halo-n and donut realizations. The halo-n and donut distributions show very good agreement, and a reduction of statistical uncertainty with growing $n$.

- Check that the effective area as a function of the true gamma-ray energy agree, within statistical uncertainties, computed for halo-n and donut realizations (see Figure 7). The halo-n and donut distributions show very good agreement, and a reduction of statistical uncertainty with growing $n$.

- We also expect the IRFs computed with the donut method to converge to those for a point like MC when we use a very narrow source morphology. In order to check this, we have produced four different donut realizations taking the expected distribution true directions morphology to be a top-hat function with radius $0.4,0.2,0.1$ and 0.05 degrees, respectively, placed at a wobble distance of $0.4^{\circ}$. Figure 8 shows the comparison of Aeff vs. Etrue between these four realizations compared to the Aeff vs. Etrue obtained from the point-like MC. Differences are smaller for smaller values of the radius, with almost perfect convergence between the $0.05^{\circ}$ radius halo and the point-like MC. 


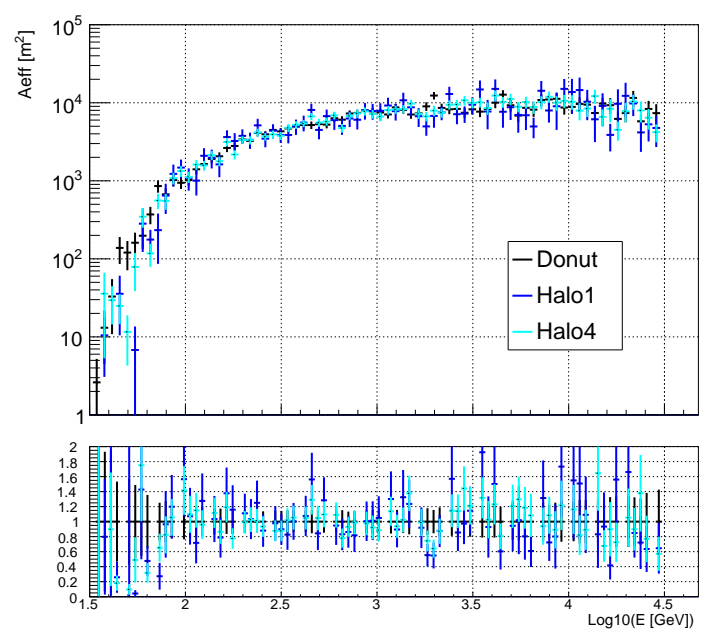

Figure 7: Comparison of Aeff (top) and Aeff ratio with respect to the donut MC (bottom) vs. Etrue computed from halo1, halo4 and donut MC. 

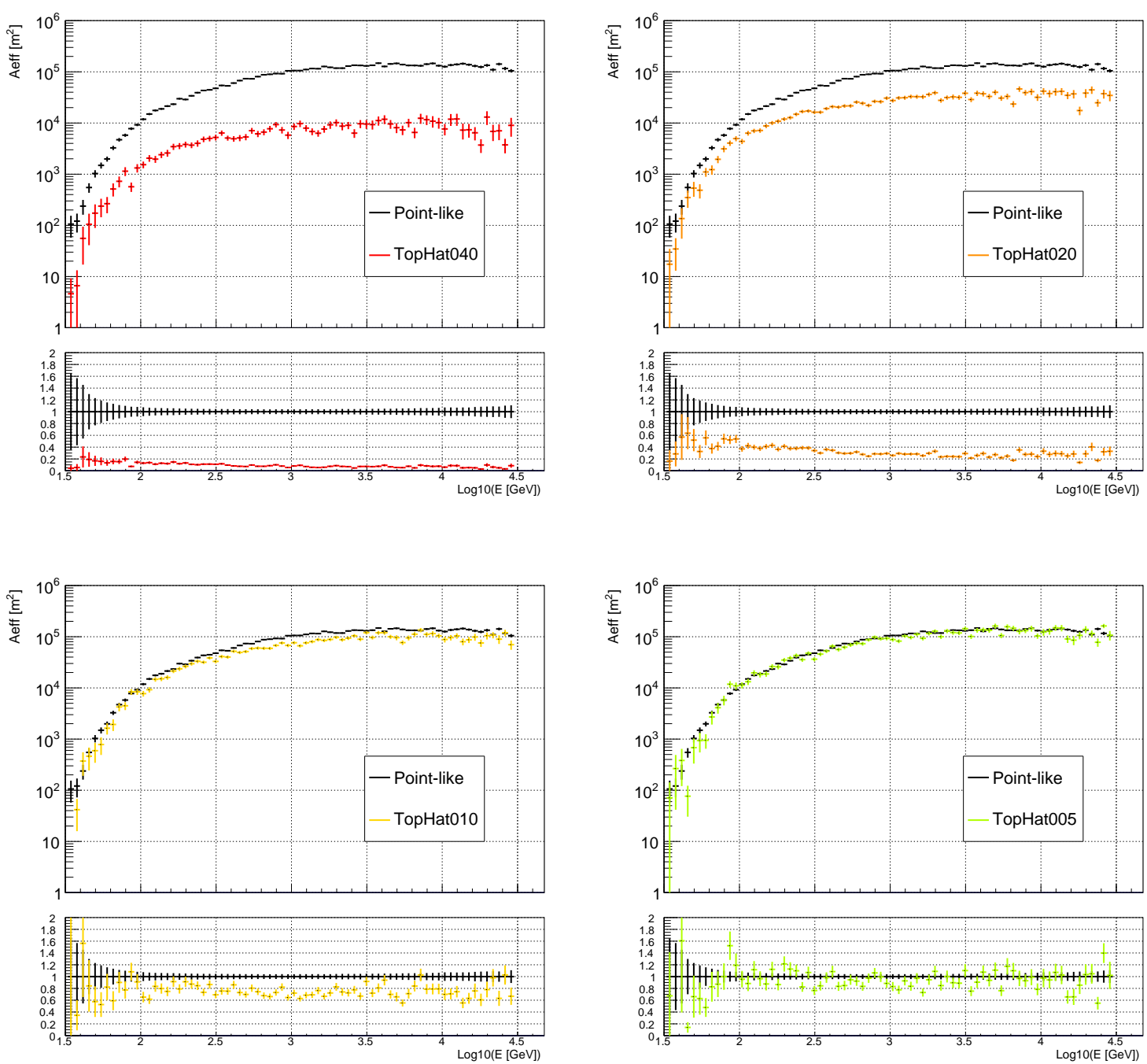

Figure 8: Comparison of Aeff (sub panel top) and Aeff ratio with respect to the point-like MC (sub panel bottom) vs. Etrue computed from donut realizations using top-hat profiles of $0.4,0.2,0.1$ and 0.05 degrees (from top bottom and left to right, respectively) and point-like MC. 